\title{
LA EXCLUSIÓN DE LOS GRANDES RIESGOS DE LOS BENEFICIOS DE LA LEY DE CONTRATO DE SEGURO EN EL PERÚ: EL PRINCIPIO DE REALIDAD*
}

\author{
THE EXCLUSION OF THE LARGE RISKS FROM \\ DE INSURANCE CONTRACT ACT IN PERU: THE \\ PRINCIPLE OF THE PRIMACY OF REALITY
}

\author{
Alonso Núñez del Prado Simons**
}

Fecha de recepción: 29 de abril de 2016 Fecha de Aceptación: 20 de mayo de 2016 Disponible en linea: 30 de julio de 2016

\section{Para Citar este articulo/To cite this article}

Núñez del Prado Simons, Alonso, La exclusión de los grandes riesgos de los beneficios de la ley de contrato de seguro en el Perú: el principio de realidad, 44 Rev.Ibero-Latinoam.Seguros, 173-182 (2016). http://dx.doi.org/10.11144/Javeriana.ris44.egrb

doi:10.11144/Javeriana.ris44.egrb

* Artículo de tipo reflexivo elaborado en el marco de la Conferencia impartida por el autor en el Congreso del Comité Ibero-Latinoamericano (CILA) de la Association Internationale de Droit des Assurances (AIDA) en La Habana, Cuba.

** Abogado, cursó el Magíster en Derecho de la Integración y el de Derecho Constitucional, es Master of Business Administration (MBA) por el College of Insurance de Nueva York, graduado en Lingüística y Literatura, además de en Filosofía, en que también cursó el Magíster. Es fundador y Director Ejecutivo del 'Observatorio de cumplimiento de planes de gobierno', profesor universitario, árbitro y conferencista internacional. Publica habitualmente en revistas jurídicas y en los principales diarios de Lima. Asimismo, es Presidente de la 'Asociación Peruana de Derecho de Seguros', Capítulo de AIDA en el Perú y director de varias entidades del sistema asegurador. Contacto: alonso.nezdelprado@gmail.com 


\section{RESUMEN}

El autor analiza el concepto de 'grandes riesgos', de origen europeo que ha sido y pretende ser mal utilizado en Latinoamérica. Defiende la alternativa legislativa por la que se optó en la ley de contrato de seguro de Perú, donde se estableció una especie de control difuso en vez del establecimiento de montos que delimiten la barrera. Critica el Proyecto de ley presentado en el Congreso peruano que pretende imitar el camino chileno.

Palabras clave: Grandes riesgos; normas indisponibles; control difuso; Solvencia II; consumidores; pequeñas empresas; interpretación contra stipulatorem.

\section{ABSTRACT}

The author analyzes the European concept of 'Large Risk', a subject which has been misunderstood in Latin America. He defend the Peruvian alternative that prefer the Judicial Review instead of the amount limits; and criticize the Project presented in the Peruvian Congress which pretends the second one, following the Chilean law that has deformed -in his opinion- the original (European) idea.

Key words: Large risk; Unavailable rules; Judicial Review; Solvency II; Consumers; Small business; Against stipulatorem interpretation. 
Para empezar quiero recordar una vez más el carácter mutual de los seguros, en estos tiempos con frecuencia relegado. En realidad las aseguradoras son administradoras de los fondos a los que aportan los asegurados y que sirven para pagar las pérdidas o siniestros de algunos de ellos, pero no es que el asegurador indemnice, por decirlo coloquialmente, 'con su plata', sino con la de todos los otros asegurados, obviamente, subdivididos por riesgos y clases de seguros. Lamentablemente este concepto básico del sistema ha sido hoy olvidado por la estructura legal de organización de las compañías de seguros como sociedades anónimas que hace parecer que son propietarias del dinero que como dije sólo administran. Hay que tener presente que ese es el fundamento y la razón de que el sistema asegurador este supervisado en todas partes del mundo. Las aseguradoras administran dinero del público y tienen que hacerlo responsablemente y de acuerdo ley.

Salvo el caso de Chile en ningún otro país de Latinoamérica se separa a los 'grandes riesgos' de la aplicación de la Ley de contrato de seguro. Las legislaciones europeas lo han hecho porque la Directiva de Solvencia II de la Unión Europea los ha obligado. Obviamente, allá el número de 'grandes riesgos' es bastante mayor y muchos de éstos son incluso propietarios de aseguradoras cautivas.

Cabe entonces preguntarnos ¿por qué en algunas legislaciones se excluyen los 'grandes riesgos' de la aplicación y beneficios de las respectivas leyes de contrato de seguro? La respuesta es bastante obvia, en esos casos la evidente asimetría entre las partes no existe o por lo menos no es tan acentuada como con los demás asegurados, sean éstos personas naturales o empresas. La razón por la que en la mayor parte de países se regula el contrato de seguro es como acabamos de señalar por el desequilibrio que se da entre asegurado y asegurador al celebrarse el contrato. Mientras el segundo es un experto en el negocio, el primero no lo conoce suficientemente, además que el último redacta el contrato (y lo va modificando y 'adecuando' en el tiempo acorde con su experiencia y conveniencia). Carece de sustento afirmar que el monto de los activos asegurados, la asesoría de brókeres y corredores de seguros, sean del nivel que fueran, ni contar con un departamento de administración de riesgos consigan equilibrar la asimetría existente en el contrato de seguros como lo demuestra el hecho de que en la inmensa mayoría de casos las pólizas de empresas importantes estén sometidas a los textos redactados por el asegurador y éstas no puedan lograr modificaciones 
significativas. Aunque lo que acabo de afirmar es válido en cualquier parte del mundo, lo es en especial en el Perú, debido al oligopolio existente en que dos compañías controlan la mayor parte del mercado. En nuestro país son verdaderamente muy pocas las empresas que pueden negociar 'de igual a igual' con las aseguradoras y lograr que las primeras modifiquen los textos pre-redactados por las últimas.

De otro lado, muchas de las normas que regulan el contrato de seguros - que se conocen en la doctrina argentina como indisponibles (artículo 158 de la Ley 17418)- son aplicables a los 'grandes riesgos', porque éstos también contratan pólizas que tienen esa condición y una exclusión tan radical carece de sustento. Resulta más conveniente un 'control difuso' y que en caso de litigio sea el juez o el árbitro el que determine qué partes de la ley de contrato de seguro son aplicables a los 'grandes riesgos' en vez de excluirlos de plano con las consecuentes desventajas que resultan de que sea una cantidad o monto establecido por el organismo supervisor el que determine cuándo el contrato está dentro del campo de aplicación de la norma. Creo que es un hecho comprobado que por este último camino resultan excluyéndose casos en los que el asegurado no ha tenido ninguna capacidad de negociación y por el contrario se ha visto obligado a aceptar las condiciones y textos pre-redactados por el asegurador, es decir que el contrato ha continuado siendo por adhesión, y tal cosa es injusta e inaceptable. El enorme poder que tienen las compañías de seguros en el mundo presiona permanentemente para evitar la regulación, tanto que en el Perú algunos funcionarios de compañías de seguros y abogados supuestamente expertos en el tema llegaron a sostener públicamente durante el proceso de aprobación de la Ley de contrato de seguro (29946), incluso en el Congreso de la República, que no era necesaria, aunque nunca pudieron responder a la contra pregunta: ¿Entonces, por qué los demás países la tienen?

Respecto a normativas el caso de Chile es un ejemplo patético, ya que allá se considera como 'gran riesgo' a la empresa que pague una prima superior a 200 unidades de fomento, es decir alrededor de US\$ 7,200.00. Considero evidente que una empresa que pague, por ejemplo, US\$ 10,000.00 de prima no pueda ser considerada como gran riesgo. Para hablar de un mercado que conozco y que no debe ser muy diferente en este aspecto al chileno, si en el Perú habláramos de primas tendríamos que pensar en un mínimo de US\$ 5 millones. Recién con una prima anual de ese volumen la empresa asegurada está en capacidad de 
negociar en igualdad de condiciones con la aseguradora. Lo expuesto sólo demuestra la gran influencia que tienen en Chile los aseguradores para haber logrado el límite indicado y que es lo que se pretende lograr aquí en el Perú y que a todas luces sería un gran abuso.

1. La directiva europea de Solvencia II (Art. 13, $\mathrm{N}^{\circ} 27$ ) que es a la que se ha adecuado España y los demás países miembros es bastante más exigente. El límite es un balance de 6.2 millones de euros, un volumen de ventas de 12.8 millones de euros y un mínimo de 250 empleados. El asegurado tiene que exceder por lo menos de dos de estos criterios para ser considerado 'gran riesgo'. En esos casos la legislación del viejo continente permite pactar incluso el sometimiento a una legislación diferente a la del domicilio de la empresa asegurada, lo que muestra que está pensada para las grandes transnacionales y sus aseguradoras cautivas, pero de ninguna manera para las empresas que paguen más de US\$7,200.00. La razón por la que las legislaciones europeas han incorporado el concepto de 'Grandes riesgos' es para permitir que las empresas que están incluidas en esa definición ${ }^{1}$ (límites mucho más exigentes que los que ha fijado Chile) puedan escoger la legislación que se les aplica. En otras palabras la razón es más bien comunitaria, es decir para facilitar la negociaciones entre empresas que tienen domicilios en diferentes países de la Unión Europea. Las diferentes leyes europeas que han incorporado el concepto no excluyen a los 'Grandes riesgos' de la aplicación de la ley, sino que le permiten adecuarse a otra. Transcribo a continuación lo expresado por el profesor de la Universidad de Milán, Pierpaolo Marano:

Mass risks, i.e. risks other than large risks, are the risks which consumers are exposed to. Therefore, their need of protection is higher than customers who "buy" the protection against large risks.

The rules, which apply to mass risks, try to allocate the jurisdiction close to the consumer. While the rules on large risks are more oriented to the "free choice" by the parties involved.

1 La directiva europea de Solvencia II (Art. 13, $\mathrm{N}^{\circ} 27$ ) que es a la que se han tenido que adecuar España y los demás países miembros es bastante más exigente que la que han impuesto los aseguradores en Chile. El límite es un balance de 6.2 millones de euros, un volumen de ventas de 12.8 millones de euros y un mínimo de 250 empleados. El asegurado tiene que exceder por lo menos de dos de estos criterios para ser considerado 'gran riesgo', pero el resultado no es que ya no estás regulado por la ley de contrato de seguro, sino que se puede escoger otra jurisdicción. 
The distinction between mass risks and large riks is "conventional", i.e. all the EU Member States agreed on such a distinction.

EU regulation on conflicts of law includes all the insurance contracts. ${ }^{2}$

Antes que la Ley de contrato de seguro entrara en vigencia en el Perú, algunos de los miembros de la Comisión que redactó el Proyecto por encargo del Congreso, vimos con estupor que en un laudo eminentes abogados - no muy conocedores de la industria- sostuvieron que la participación de un bróker o corredor de seguros equilibraba el contrato hasta el punto de que ya no podía tenerse como celebrado por adhesión, cuando en el caso que resolvían todo el texto y cláusulas adicionales no habían sufrido modificación y eran las pre-redactadas por el asegurador. En otras palabras, no tuvieron claro cuándo un contrato es por adhesión.

En un claro intento de retroceder lo andado, en el Congreso de la República se preparó un Anteproyecto (Juan Carlos Eguren) que pretendía modificar la Ley de contrato de seguro (29946) que fue promulgada el 27 de noviembre de 2012 y que entró en vigencia el 26 de mayo de 2013, es decir que todavía no ha cumplido dos años de aplicación. El Anteproyecto, que felizmente no obtuvo el consenso de la bancada, sostenía que el texto actual (Artículo III) era insuficiente porque no excluía de la aplicación de la norma a los 'grandes riesgos', y con esta disculpa se pretendía contrabandear la limitación de la aplicación de la norma sólo a personas naturales y micro y pequeñas empresas (MYPES), pero no a las medianas y más grandes, estableciendo un monto (activos o primas) que fijaría el ente supervisor a partir del cual la ley no se aplicaría.

En ningún país la Ley de contrato de seguro está expresamente limitada a amparar sólo a las personas naturales y a las MYPES, como ocurre, parcialmente, con nuestro Código de Protección al Consumidor que en verdad incluye a empresas más grandes siempre que se adecúen a la definición de consumidor ${ }^{3}$.

2 Mensaje electrónico que me enviara el día 6 de agosto de 2015. Traducido al español sería: Los riesgos masivos, por ejemplo los riesgos diferentes a los grandes riesgos, son los riesgos a los que están expuestos los consumidores. En consecuencia, su necesidad de protección es mayor que los consumidores que 'compran' la protección contra grandes riesgos. Las reglas que se aplican a los riesgos masivos, intentan fijar la jurisdicción más cercana al consumidor. Mientras que las reglas en grandes riesgos están orientadas a que las partes involucradas la escojan. La distinción entre riesgos masivos y grandes riesgos es 'convencional'. Por ejemplo todos los estados miembros de la Unión Europea aceptaron esa distinción. La regulación de la Unión Europea en conflictos de leyes incluye todos los contratos de seguros.

3 1. Consumidores o usuarios 1.1 Las personas naturales o jurídicas que adquieren, utilizan o disfrutan como destinatarios finales productos o servicios materiales e inmateriales, en beneficio propio o de 
La argumentación que usan resulta engañosa. Afirman que a las empresas que se excluiría serían a las denominadas 'grandes riesgos' que supuestamente tienen la capacidad de negociar de igual a igual con las aseguradoras, sin embargo, como ha ocurrido en Chile, el monto resulta siendo pequeño y se excluye de los beneficios de protección de la ley a un importante número de firmas que en realidad no tienen ninguna posibilidad de negociar con las aseguradoras en igualdad de condiciones y no les queda más remedio que aceptar sus imposiciones, más en un mercado como el peruano donde hay dos empresas que lo dominan.

Cuando fue consultada por el Congreso antes de la aprobación y promulgación de la mencionada ley, la Superintendencia de Banca, Seguros y AFP (SBS) -copiando la ley española, como lo hizo en la mayor parte de sus sugerencias- había propuesto la exclusión de los 'grandes riesgos' del campo de aplicación de la ley, pero en la Comisión que redactó el Proyecto optamos por una alternativa diferente, por la enorme influencia que tienen los aseguradores en esta institución en el Perú, y que probablemente resultaría en límites muy bajos -como ocurre en el país del sur- que además no se actualizarían oportunamente, terminando por excluir a un importante número de negocios asegurados de los beneficios de la ley.

La solución escogida por la Ley 29946 es remitirse a las pólizas, ya que los otros criterios, como el volumen de activos, montos de primas o número de empleados, pueden ser engañosos. Entonces cuando se pregunta ¿con qué criterio se haría el 'control difuso' respecto de los grandes riesgos?, la respuesta es que -como lo establece el artículo III de la Ley 29946- el criterio es el texto de la póliza ('wording' en inglés). Si éste es el redactado por el asegurador, significa que el contrato se ha realizado por adhesión y tiene que tener todas las protecciones de la ley. Si por el contrario contiene cambios, el juez o árbitro tendrá que decidir sobre la importancia de éstos y resolver hasta donde corresponde considerar el contrato como de adhesión y por tal también hasta donde debe contar con las protecciones de la ley. Este camino evita que haya sociedades que resulten no amparadas por la ley, pero que en la realidad no estén en capacidad de cambiar ni una coma del contrato redactado por el asegurador.

su grupo familiar o social, actuando así en un ámbito ajeno a una actividad empresarial o profesional. No se considera consumidor para efectos de este Código a quien adquiere, utiliza o disfruta de un producto o servicio normalmente destinado para los fines de su actividad como proveedor. (Artículo IV.- Definiciones del Código de Protección y Defensa del Consumidor, Ley 29571) 
A pesar de que se ha explicado públicamente la forma de limitar la aplicación de nuestra ley a los 'grandes riesgos', los aseguradores consiguieron que la SBS apoyara mediante un oficio el Ante-Proyecto de excluirlos totalmente mediante una norma modificatoria que, felizmente como he señalado, no prosperó ni siquiera en la bancada del congresista que la promovía y que por ahora ha quedado en nada, aunque no tengo duda que las compañías insistirán y de no conseguirlo intentarán pasarla por algún otro canal.

Teniendo en cuenta que tal como quedó al final la ley en el Perú salvo en los seguros personales, obligatorios y masivos las pólizas son redactadas y también modificadas por las aseguradoras sólo registrándolas en la SBS, en la Comisión consideramos que la mejor manera de afrontar el problema era la que he expuesto y que materializamos en el ya mencionado artículo III de las Disposiciones Generales (Título I) de la Ley 29946 donde establecimos que:

"El contrato de seguro se celebra por adhesión, excepto en las cláusulas que se hayan negociado entre las partes y que difieran sustancialmente con las pre redactadas".

Como se puede ver en vez de guiarnos por el balance, volumen de ventas, número de empleados, sumas aseguradas o primas esta alternativa se remite a la realidad, el texto objetivo de la póliza. En él se puede ver si efectivamente el asegurado (empresa grande o pequeña) ha sido capaz de negociar de igual a igual con la compañía y cambiar el condicionado pre-redactado por la aseguradora. En otras palabras, por este camino no hay duda y en el caso de que el asegurador sostenga que el asegurado 'negoció de igual a igual' tiene que demostrarlo con las modificaciones que logró al redactar el contrato. En el Perú, como ya he indicado las empresas que logran que las aseguradoras cambien los términos pre-redactados se pueden contar con los dedos de la mano y en esos casos les resultará muy fácil demostrarlo. Conviene dejar claro que es usual que se incluyan 'cláusulas' que modifican las 'condiciones generales', pero con las condiciones especiales también pre-redactadas por la compañía, aunque a veces éstas aceptan pequeñas modificaciones que son los textos preparados por los grandes corredores para todos sus clientes y que buscan diferenciarlos. Como es de suponer tampoco las 'condiciones particulares' (nombre y suma asegurados, etc.) que individualizan el riesgo, pueden considerarse como importantes modificaciones que le quiten la calidad de contrato por adhesión a una póliza. 
Consideramos que este camino constituía una mejor opción legislativa porque además no excluye a los 'grandes riesgos' de la aplicación de ley, sino que sólo aligera la 'interpretación contra stipulatorem' y deja a criterio del juez o árbitro la decisión de cómo aplica los beneficios de la norma, además de que permite separar las cláusulas 'negociadas' de las 'pre-redactadas' y no excluir de los beneficios de la ley a un asegurado que eventualmente pudo haber logrado pactar alguna cláusula aislada o que, de haberse optado por el otro camino, excediera los límites fijados por la institución supervisora, aun cuando no hubiera tenido ninguna posibilidad de negociar los términos y condiciones de su póliza. Tiene también la virtud de de separar las diferentes pólizas en un mismo cliente, ya que éste podría tener pólizas en los que ha logrado negociar 'de igual a igual', pero otras en que esto ha sido imposible por las condiciones del mercado.

Como era de suponer, la opción que escogimos en la Comisión no es del agrado de los aseguradores y por eso están intentando hacer modificaciones. Al final el problema es que la nueva ley les ha restado poder y ahora ya no pueden hacer y deshacer como antes, sino que tienen que someterse a algunas reglas que, afortunadamente, son protectoras del asegurado porque, como he indicado y todos sabemos, el contrato de seguro es asimétrico. Una de las partes es la que conoce del negocio y la que redacta el contrato. Además, con el tiempo lo va 'afinando' y haciendo exclusiones y creando restricciones que muchas veces reducen los límites de cobertura.

Otro argumento que las aseguradoras han esgrimido es la dificultad que les causa la ley 29946 en la negociación de reaseguros. Cabe preguntarles ¿por qué los demás países latinoamericanos no tienen ese problema?, y ¿por qué los europeos -que tienen leyes más protectoras de los asegurados que la nuestra- tampoco los tienen? En la industria hay que entender a los reaseguradores como proveedores de las aseguradoras; en consecuencia el público, es decir los asegurados, no tiene por qué ser afectado por las condiciones que éstas consigan. Esa es la razón por la que el artículo 322 de la Ley (26702) General del Sistema Financiero y orgánica de la Superintendencia de Banca, Seguros y AFP's (SBS) establece que existe completa independencia entre los contratos de seguros y reaseguros. De otro lado, es relevante recordar que, salvo excepciones, los reaseguradores se someten a las leyes de los países de las compañías que reaseguran; es más, hay casos (Ejem. Brasil) en 
los que por ley los propios contratos de reaseguro están sometidos a la jurisdicción y las leyes del país del domicilio de la compañía reasegurada. Algo que resulta incómodo en reaseguros -y que las aseguradoras locales hacen lo posible por evitar y casi nunca aceptan- es que el reasegurador imponga una jurisdicción y legislación extranjeras para el contrato, lo que obliga a que en caso de conflicto la aseguradora tenga que ir a litigar en otro país. Esto pudo evitarse si se hubiera incluido en la ley el artículo que obligaba a que los contratos de reaseguros estuvieran sometidos a la ley y jueces peruanos, pero que fue eliminado porque la SBS, equivocadamente, creía que la mayor parte de contratos facultativos están sujetos a leyes y tribunales extranjeros, cuando esto, más bien, rara vez ocurre ${ }^{4}$

\section{BIBLIOGRAFÍA}

Bickelhaup, David. General Insurance. Richard D. Irwin Inc. Illinois, 1983.

Bustamante Ferrer, Jaime.- Manual de Principios Jurídicos del Seguro.- Editorial Temis.- Bogotá, 1983.

Curso de seguros.- Chartered Insurance Institute.- Elementos de Seguros.- Editorial Mapfre.- Madrid, 1973.

Efrén Ossa, G. J.- Teoría General del Seguro.- Editorial Temis.- Bogotá, 1984.

Efrén Ossa, G. J.- Tratado Elemental de Seguros.- Editorial Leonor.- Bogotá, 1963.

Halperin, IsaAc.- Lecciones de Seguros.- Ed. Depalma.- Buenos Aires, 1983.

LORIMER, JAMES y otros.- The Legal Environment of Insurance.- American Institute for Property and Liability Underwriters Inc.- V Pensylvania, 1981 (2da. Edic.)

MeHr, Robert I.- Fundamentals of Insurance.- Richard D. Irwing, Inc.- Illinois, 1983.

Soler Aleu, Amadeo.- El Nuevo Contrato de Seguros.- Ed. Astrea.- Buenos Aires, 1978.

Stiglitz, RubÉn. S. El Siniestro. Ed. Astrea, Buenos Aires, 1980.

Stiglitz, RubÉn S.- Contrato de Seguros.- La Ley.- Buenos Aires, 2004.

4 He consultado personalmente con los principales brókeres de reaseguros (Marsh, Aon, JLT, Guy Carpenter, Willis, etc.) y me lo han confirmado. 